\title{
Hyperplasie épithéliale focale et syndrome de Waldmann. Présentation d'un cas
}

\author{
Marc El Hage ${ }^{1 \star}$, Semaan Abi Najm ${ }^{1}$, Tommaso Lombardi ${ }^{2}$, Jacky Samson ${ }^{1}$ \\ 1 Division de Stomatologie et Chirurgie orale, Faculté de Médecine, Genève, Suisse \\ 2 Laboratoire d'Histopathologie buccale, Faculté de Médecine, Genève, Suisse
}

(Med Buccale Chir Buccale 2008;14:233-8)

\author{
Mots clés : \\ hyperplasie épithéliale \\ focale / \\ papillomavirus / \\ lymphangiectasie \\ intestinale / \\ lymphome MALT
}

\section{Key words:}

focal epithelial hyperplasia / papillomavirus /

intestinal lymphangiectasia /

MALT lymphoma

\begin{abstract}
Résumé - L'hyperplasie épithéliale focale (HEF) ou maladie de Heck est une affection virale tumorale bénigne qui touche la muqueuse buccale chez les enfants et les adultes jeunes. Elle est due à un VPH (virus des papillomes humains) de types 13 ou 32. Un cas d'HEF a été observé chez une patiente de 20 ans atteinte d'une lymphangiectasie intestinale, ou syndrome de Waldmann, et d'un lymphome MALT duodénal. Le déficit immunitaire induit par le syndrome de Waldmann a sans doute favorisé le développement du lymphome MALT et de l'HEF. Ceci expliquerait leur évolution synchrone.
\end{abstract}

\begin{abstract}
Focal epithelial hyperplasia and Waldmann syndrome: case report. Focal epithelial hyperplasia (FEH) or Heck disease is a viral benign pathology affecting the oral mucosa of children and young adults. It is caused by human papillomavirus types 13 or 32 . The case of a 20-year-old woman with FEH, Waldmann syndrome and a MALT lymphoma is described. The immunodeficiency resulting from the Waldmann syndrome induced the occurrence of the MALT lymphoma and the FEH. This would explain their parallel evolution.
\end{abstract}

L'hyperplasie épithéliale focale (HEF) ou maladie de Heck est une affection virale tumorale bénigne qui touche la muqueuse buccale, décrite initialement chez des enfants et des adultes jeunes appartenant à certaines ethnies (esquimaux, amérindiens...). Puis elle a été observée dans d'autres ethnies et chez des sujets présentant un déficit immunitaire acquis. Dans le cas rapporté, l'HEF est associée au syndrome de Waldmann et à un lymphome MALT (Mucosa-Associated Lymphoid Tissue).

\section{Observation}

M.0.S. est une jeune Somalienne, née en 1982, qui consulte en février 2002 pour des lésions buccales multiples, non douloureuses. Depuis l'enfance elle présentait des œdèmes cycliques des membres inférieurs, des lésions verruqueuses cutanées et génitales qui ont conduit à la découverte d'un syndrome déficitaire dissocié, comportant une hypoalbuminémie majeure, une lymphopénie, une carence en vitamine D

^Correspondance : Marc El Hage, marc.elhage@medecine.unige.ch et une hypotriglycéridémie. Ces symptômes ont fait suspecter une entéropathie exsudative chronique; la lymphopénie et la baisse dissociée des triglycérides (dont la lymphe digestive est très riche) ont fait évoquer une lymphangiectasie intestinale ou syndrome de Waldmann, maladie orpheline congénitale qui se traduit par une entéropathie exsudative avec un déficit immunitaire secondaire à la perte de lymphocytes. Le diagnostic définitif a été établi en 1999. De plus, une œsophago-gastro-duodénoscopie faite en 2006, a révélé la présence de plages duodénales blanchâtres ayant un aspect histopathologique compatible avec celui d'un lymphome MALT.

Chez cette patiente, le déficit immunitaire n'entraîne pas d'infections opportunistes hormis des infections dues aux virus des papillomes humains (VPH) : verrues vulgaires sur le revêtement cutané et condylomes acuminés dans la région anogénitale. A l'examen endo-buccal, on observe sur la muqueuse jugale, la muqueuse labiale et la demi-muqueuse labiale, de nombreuses lésions papuleuses bien limitées, mollasses, dont la taille varie de quelques millimètres à plus d'un centimètre (Figs. 1 et 2); ces lésions s'effacent lors de la mise en tension des tissus. Ce tableau clinique fait suspecter une HEF. 

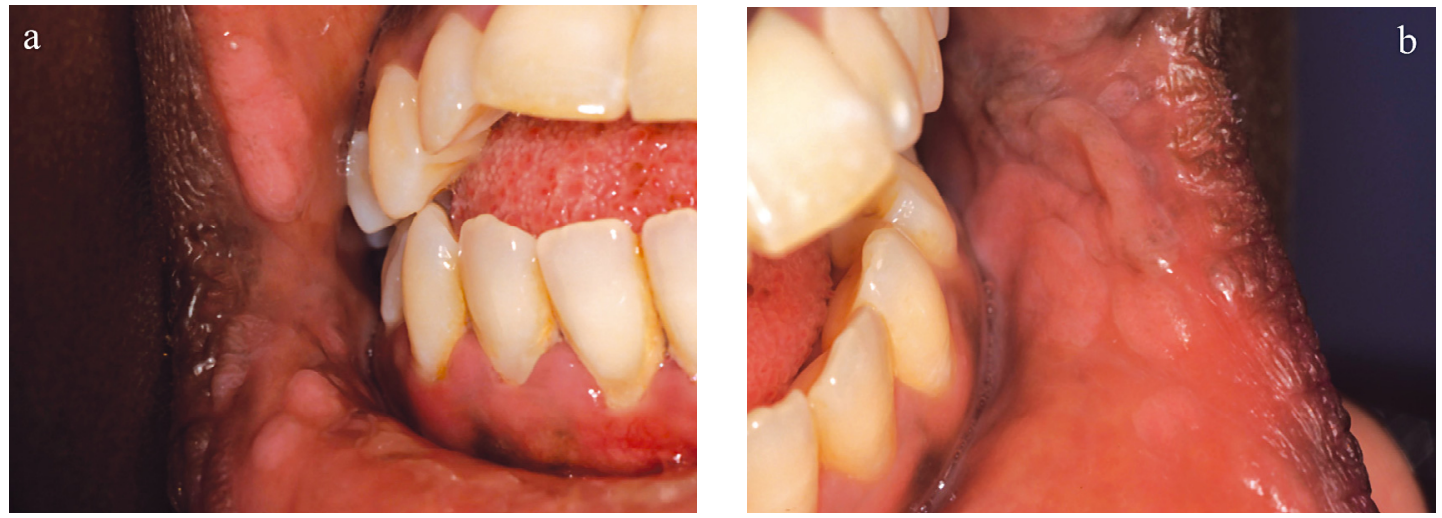

Fig. 1. Nombreuses lésions sur la muqueuse labiale droite (a) et gauche (b). Fig. 1. Numerous lesions on the right (a) and left (b) labial mucosa.

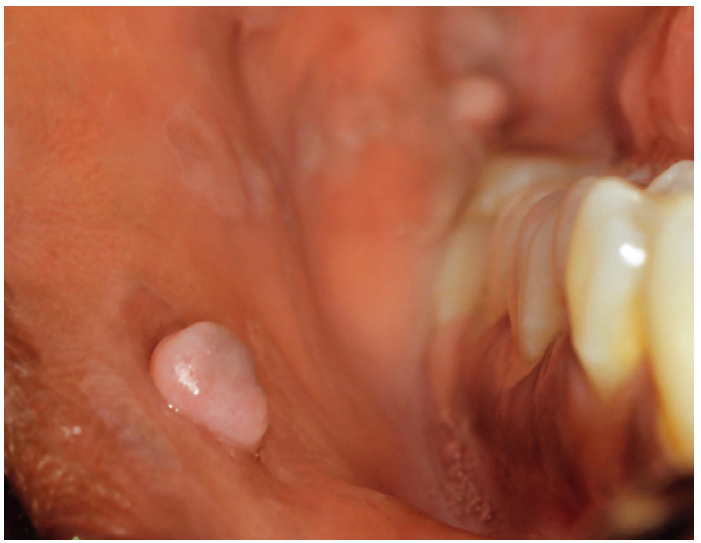

Fig. 2. Lésion isolée sur la muqueuse jugale. Fig. 2. Unique lesion on the buccal mucosa.

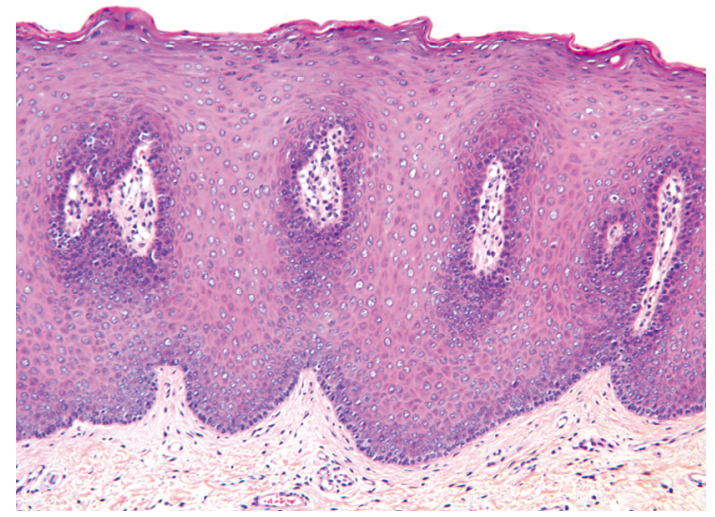

Fig. 3. Crêtes épithéliales allongées et anastomosées. Fig. 3. Elongated and anastomosed rete ridges.

L'examen histopathologique effectué sur deux lésions labiales inférieures a confirmé le diagnostic d'HEF. La surface épithéliale comporte 1 à 3 assises de cellules parakératosiques et il existe une acanthose avec des crêtes épithéliales allongées et anastomosées (Fig. 3). On note la présence de quelques koïlocytes et de rares pseudo-mitoses (Fig. 4). La polymerase chain

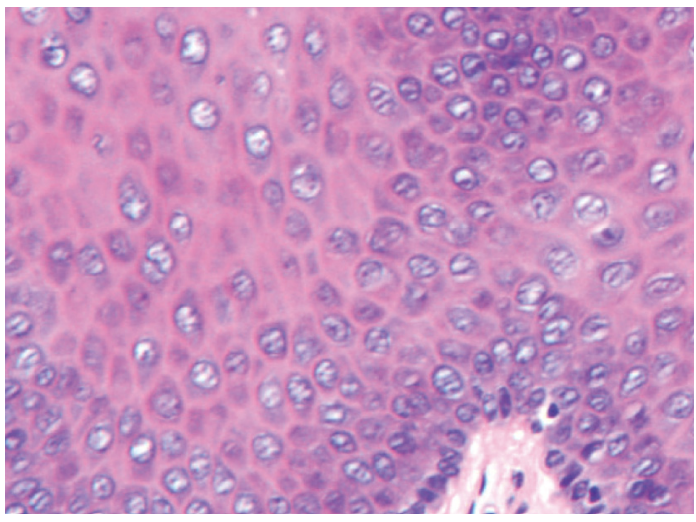

Fig. 4. Présence de quelques koïlocytes et de rares pseudo-mitoses. Fig. 4. Some koilocytes and very few pseudomitosis cells are present.

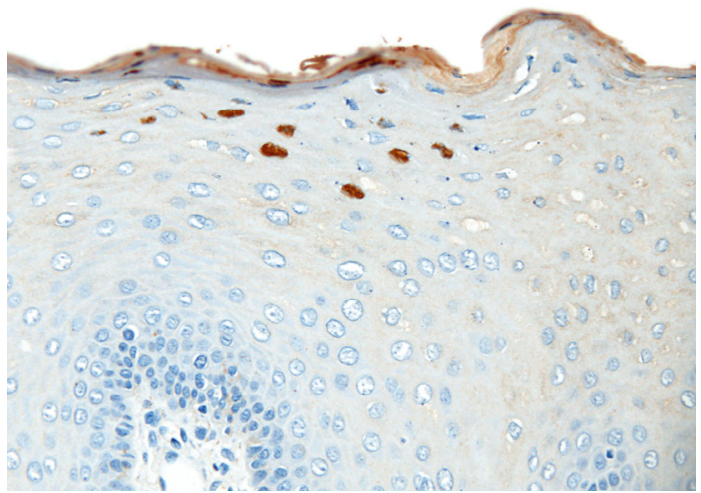

Fig. 5. Marquage positif de quelques cellules pour le VPH. Fig. 5. Some cells are labelled by the antibody against HPV.

reaction (PCR) confirme la présence de VPH, le séquençage et une analyse Basic Local Alignment Search Tool (BLAST) montrent qu'il s'agit d'un VPH de type 13 (Fig. 5).

Chez cette patiente, il existe manifestement un lien étroit entre l'état immunitaire et les manifestations cliniques de l'HEF. Pour traiter la lymphangiectasie intestinale, elle reçoit 

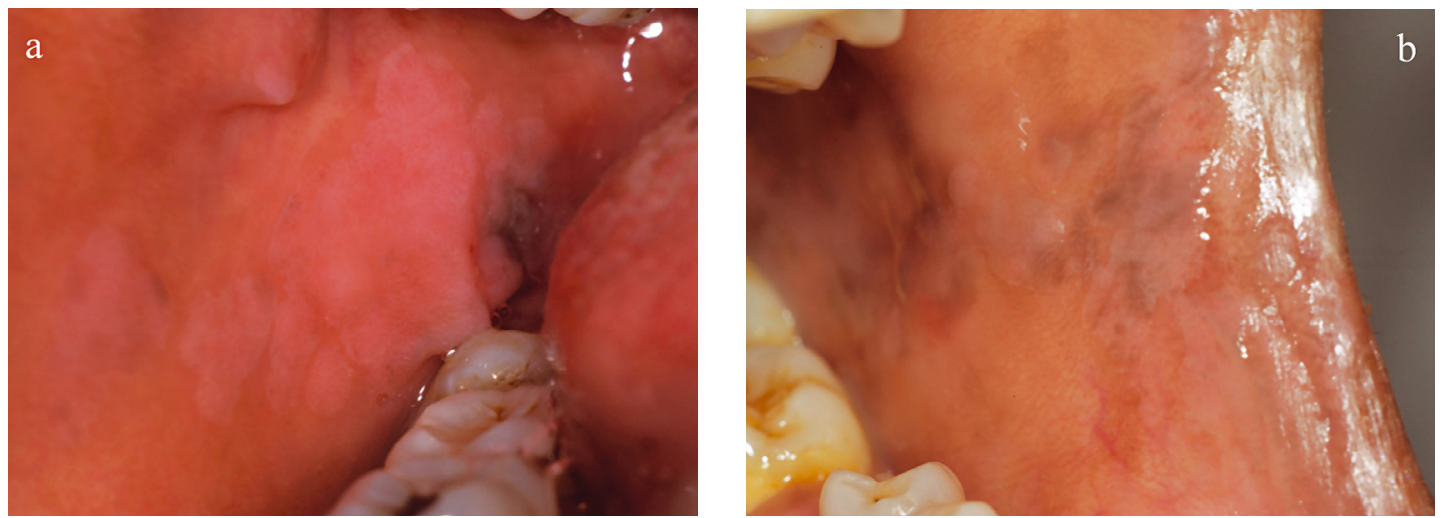

Fig. 6. Disparition complète des lésions labiales et jugales droites (a) et gauches (b).

Fig. 6. Total disappearance of the right (a) and left (b) cheek lesions.

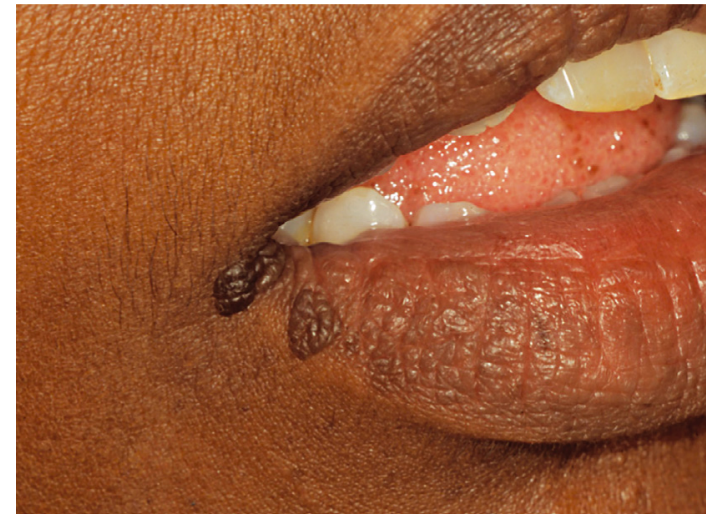

Fig. 7. Deux nouvelles lésions siégeant sur la demi-muqueuse, débordant un peu sur le revêtement cutané.

Fig. 7. Two new mucous lesions cross the mucocutaneous junction.

une injection intraveineuse hebdomadaire d'albumine. Ces injections entraînent une diminution de la perte des lymphocytes et donc une diminution du déficit immunitaire. La patiente étant peu compliante, son état immunitaire varie constamment et elle se trouve souvent en situation de déficit immunitaire. Ceci semble se refléter sur les manifestations cliniques de l'HEF. En effet, lors des visites de contrôle, l'examen endo-buccal montre une variation de la taille et du nombre des lésions de l'HEF, parallèles à l'importance du déficit immunitaire de la patiente. Depuis quelques mois, le traitement a été modifié (meilleure adaptation des doses d'albumine) et la patiente est plus compliante : ceci a entrainé la disparition de toutes les lésions buccales et génitales (Fig. 6).

Deux lésions sont apparues récemment près de la commissure labiale droite sur la demi-muqueuse labiale inférieure, débordant un peu sur le revêtement cutané (Fig. 7). Comme leur aspect ne ressemble pas à celui des verrues vulgaires, il s'agit probablement de lésions d'HEF. Bien que la patiente souhaite leur exérèse pour des raisons esthétiques, il lui a été proposé d'attendre pour mieux apprécier l'évolution. Cette mesure de précaution vise à retarder tout geste chirurgical susceptible d'entraîner le développement d'une cicatrice disgracieuse ou d'une chéloïde.

\section{Discussion}

Les sujets immunodéficients développent plus facilement certaines tumeurs malignes, plus particulièrement des lymphomes. Une étude faite par Kersy et al. (1988) montre que $48.6 \%$ des processus malins survenant chez des individus souffrant d'une immunodéficience sont des lymphomes nonhodgkiniens [1].

Le déficit immunitaire induit par la lymphangiectasie intestinale semble, comme les autres déficits immunitaires, favoriser les lymphomes digestifs qui surviennent assez tardivement [2]. Le lymphome MALT se développe à partir des lymphocytes du tube digestif, surtout quand ils sont soumis à une stimulation inflammatoire ou auto-immune chronique $[3,4]$. Pour cette raison, plusieurs endoscopies de dépistage ont été effectuées à partir de 2001. En 2006, une œsophago-gastroduodénoscopie montre la présence de plages duodénales blanchâtres, dont l'aspect histopathologique est compatible avec celui d'un lymphome MALT associé à une population lymphocytaire B. Un nouveau bilan endoscopique complet réalisé en février 2007 n'a pas retrouvé de lésions macroscopiques lymphomateuses, alors que le même clone lymphocytaire $B$ était toujours présent. En conclusion, il s'agissait d'un lymphome MALT duodénal de stade très précoce, associé à un syndrome de Waldmann. Les lésions duodénales qui étaient inconstantes depuis la découverte initiale (2006), ont régressé de façon complète avec la disparition du déficit immunitaire.

L'HEF ou maladie de Heck est une affection virale tumorale bénigne qui touche la muqueuse buccale chez les enfants et les adultes jeunes. On considère qu'elle a été décrite pour la première fois par Heck et al. en 1965 [5]. En réalité, dans le même numéro de la même revue, l'article de Heck et al. est suivi par un article de Witkop et al. qui décrivent la même entité [6]. Sa prévalence exacte n'est pas connue puisque la plupart des publications rapportent des cas isolés et seulement quelques publications comportent un grand 
nombre de patients $[7,8]$. L'HEF est due à des VPH de types 13 ou $32[9,10]$. Elle se traduit par de multiples papules ou nodules indolores, de nombre et de taille variables, mesurant de 0.1 à $1 \mathrm{~cm}$, pouvant intéresser l'ensemble de la muqueuse buccale, mais le plus souvent uniquement les lèvres et les joues. Elles persistent pendant plusieurs années ou régressent tandis que d'autres se développent.

Le traitement de l'HEF n'est pas bien codifié, et il existe plusieurs modalités de traitement : exérèse chirurgicale des lésions, cryochirurgie, thérapie au laser diode et utilisation de certains agents topiques $[11,12]$. Les facteurs qui déterminent la susceptibilité pour l'HEF et sa transmission sont encore mal connus, mais cette affection touche de préférence certains groupes ethniques; la présence de plus d'un cas d'HEF dans une même famille suggère qu'une prédisposition génétique intervient dans la pathogénie de cette maladie [5-7, 13-16]. Un facteur génétique présent dans certaines ethnies (esquimaux, amérindiens, turcs, nord-africains...), probablement responsable d'un déficit immunitaire primaire, mineur, non identifié, semble favoriser le développement de l'affection le plus souvent dans l'enfance. Une hypothèse émise par García-Corona et al. [17] suggère que le locus HLA-DR joue un rôle dans la physiopathologie de l'HEF en sélectionnant les individus génétiquement prédisposés, particulièrement ceux qui sont homozygotes pour HLA-DR4 et son allèle 0404.

Après sa description chez des sujets appartenant à certaines ethnies susceptibles de présenter un déficit immunitaire mineur, l'HEF a été observée chez des patients immunodéprimés : sujets séropositifs pour le VIH, sujets transplantés recevant un traitement immunosuppresseur $[18,19]$. Certains déficits immunitaires semblent donc favoriser l'infection par les VPH de types 13 et 32 [20]. Le déficit immunitaire induit par le syndrome de Waldmann constitue probablement un autre type de déficit immunitaire favorisant l'HEF.

\section{Références}

1. Kersey JH, Shapiro RS, Filipovich AH. Relationship of immunodeficiency to lymphoid malignancy. Pediatr Infect Dis J 1988;7:S10-2.

2. Bouhnik Y, Etienney I, Nemeth J, Thevenot T, Lavergne-Slove A, Matuchansky C. Very late onset small intestinal B cell lymphoma associated with primary intestinal lymphangiectasia and diffuse cutaneous warts. Gut 2000;47:296-300.

3. Inagaki H. Mucosa-associated lymphoid tissue lymphoma: molecular pathogenesis and clinicopathological significance. Pathol Int 2007;57:474-84.

4. Aghamohammadi A, Parvaneh N, Tirgari F, Mahjoob F, Movahedi M, Gharagozlou M, Mansouri M, Kouhi A, Rezaei N, Webster D. Lymphoma of mucosa-associated lymphoid tissue in common variable immunodeficiency. Leuk Lymphoma 2006;47:343-6.
5. Archard HO, Heck JW, Stanley HR. Focal epithelial hyperplasia: an unusual oral mucosal lesion found in Indian children. Oral Surg Oral Med Oral Pathol 1965;20:201-12.

6. Witkop CJ Jr, Niswander JD. Focal epithelial hyperplasia in Central and South American Indians and Latinos. Oral Surg Oral Med Oral Pathol 1965;20:213-17.

7. Carlos BR, Sedano HO. Multifocal papilloma virus epithelial hyperplasia. Oral Surg Oral Med Oral Pathol 1994;77:631-5.

8. Matute TG, Gonzalez PLV, Acosta OE. Prevalencia de hiperplasia epitelial focal en escolares de la comunidad indigena de Cristania, Municipio de Jardin, Antioquia, 1998. Rev Fac Odontol Univ Ant 1999;11:15-9.

9. Pfister H, Hettich I, Runne U, Gissmann L, Chilf GN. Characterization of human papilloma virus type 13 from focal epithelial hyperplasia Heck lesions. J Virol 1983;47:363-6.

10. Padayechee A, van Wyk CW. Human papillomavirus (HPV) DNA in focal epithelial hyperplasia by in situ hybridization. J Oral Pathol Med 1991;20:210-4.

11. Luomanen M. Oral focal epithelial hyperplasia removed with $\mathrm{CO} 2$ laser. Int J Oral Maxillofac Surg 1990;19:205-7.

12. Cohen PR, Hebert AA, Adler-Storthz K. Focal epithelial hyperplasia: Heck disease. Pediatr Dermatol 1993;10:245-51.

13. Garlick JA, Calderon S, Buchner A, Mitrani-Rosenbaum S. Detection of human papillomatosis (HPV) DNA in focal epithelial hyperplasia. J Oral Pathol Med 1989; 18:172-7.

14. Premoli-De-Peroco G, Cisternas JP, Ramìrez JL, Galindo I. Focal epithelial hyperplasia: human-papillomavirus-induced disease with a genetic predisposition in a Venezuelan family. Hum Genet 1993;91:386-8.

15. Gomez A, Calle A, Arcila G, Pindborg JJ. Focal epithelial hyperplasia in a halfbreed family of Colombians. J Am Dent Assoc 1969;79:663-7.

16. Schock RK. Familial focal epithelial hyperplasia: report of a case. Oral Surg Oral Med Oral Pathol 1969;28:598-602.

17. García-Corona C, Vega-Memije E, Mosqueda-Taylor A, YamamotoFurusho JK, Rodríguez-Carreón AA, Ruiz-Morales JA, Salgado N, Granados J. Association of HLA-DR4 (DRB1*0404) with human papillomavirus infection in patients with focal epithelial hyperplasia. Arch Dermatol 2004;140:1227-31.

18. Vilmer C, Cavelier-Balloy B, Pinquier L, Blanc F, Dubertret L. Focal epithelial hyperplasia and multifocal human papillomavirus infection in an HIV-seropositive man. J Am Acad Dermatol 1994;30:497-8.

19. Viraben R, Aquilina C, Brousset P, Bazex J. Focal epithelial hyperplasia (Heck disease) associated with AIDS. Dermatology 1996;193: 261-2.

20. Moerman M, Danielides VG, Nousia CS, Van Wanzeele F, Forsyth $\mathrm{R}$, Vermeersch $\mathrm{H}$. Recurrent focal epithelial hyperplasia due to HPV13 in an HIV-positive patient. Dermatology 2001;203: 339-41. 